\title{
MR imaging of the foot and ankle: patterns of bone marrow signal abnormalities
}

\author{
Weishaupt, Dominik ; Schweitzer, Mark E
}

\begin{abstract}
Diagnosis of marrow disorders of the foot and ankle is among the more challenging aspects of MR interpretation. Evaluation of normal and abnormal bone marrow with regard to pattern, distribution, and signal characteristics on different sequences often allows a specific diagnosis. This pictorial review illustrates MR imaging findings of normal variants of bone marrow of the foot and ankle, and the varied responses of bone marrow to trauma, stress, or disease
\end{abstract}

DOI: https://doi.org/10.1007/s003300101070

Posted at the Zurich Open Repository and Archive, University of Zurich

ZORA URL: https://doi.org/10.5167/uzh-156256

Journal Article

Published Version

Originally published at:

Weishaupt, Dominik; Schweitzer, Mark E (2002). MR imaging of the foot and ankle: patterns of bone marrow signal abnormalities. European Radiology, 12(2):416-426.

DOI: https://doi.org/10.1007/s003300101070 
Dominik Weishaupt Mark E. Schweitzer

\section{MR imaging of the foot and ankle: patterns of bone marrow signal abnormalities}

Received: 10 April 2001

Revised: 21 June 2001

Accepted: 26 June 2001

Published online: 28 July 2001

(C) Springer-Verlag 2001

D. Weishaupt $(\bowtie)$

Institute of Diagnostic Radiology,

University Hospital Zurich,

Raemistrasse 100, 8091 Zurich, Switzerland

E-mail: dominik.weishaupt@dmr.usz.ch

Phone: +41-1-2553059

Fax: +41-1-2554443

M.E. Schweitzer

Department of Radiology,

Thomas Jefferson University Hospital,

Philadelphia, PA, USA

\begin{abstract}
Diagnosis of marrow disorders of the foot and ankle is among the more challenging aspects of MR interpretation. Evaluation of normal and abnormal bone marrow with regard to pattern, distribution, and signal characteristics on different sequences often allows a specific diagnosis. This pictorial review illustrates MR imaging findings of normal variants of bone marrow of the foot and ankle, and the varied responses of bone marrow to trauma, stress, or disease.
\end{abstract}

Keywords MR imaging $\cdot$ Bone marrow $\cdot$ Foot $\cdot$ Ankle

\section{Introduction}

Magnetic resonance imaging has demonstrated its usefulness in assessment of normal and abnormal bone marrow. Using MR imaging the continuous change of normal bone marrow composition throughout life and the varied responses of bone marrow to trauma or disease can be monitored [1].

Magnetic resonance imaging characterization of location, morphology, distribution, and signal characteristics of a bone marrow abnormality enables diagnosis of a wide range of foot and ankle disorders with a high level of specificity. This pictorial review highlights applications of MR imaging of marrow disorders of the foot and ankle including traumatic and stress-related marrow changes, avascular necrosis, osteochondral defects, arthritic, and neuropathic arthropathies as well as osteomyelitis.

\section{Technical considerations}

The MR appearance of bone marrow is influenced by both composition (fat, water, minerals) and the specific imaging sequence used [1]; however, most of the MR imaging signal characteristics of marrow are dependent on the fat content $[2,3]$. A routine MR evaluation of bone marrow of the foot and ankle should include a T1weighted spin-echo sequence and a fat-suppressed T2weighted fast spin-echo or short tau inversion recovery (STIR) sequence. Since cellular infiltration within the fatty marrow may be less conspicuous or entirely obscured on T2-weighted fast spin-echo sequences, use of some form of fat suppression is mandatory on T2weighted fast spin-echo MR images [4, 5]. The most widely used form of fat suppression is frequency-selective presaturation. With this method, however, local field inhomogeneities that arise in areas of significantly varying morphology (e.g., ankle) often result in an uneven fat suppression. The inhomogeneous fat suppres- 
sion may result in areas of apparent increased signal intensity on T2-weighted fast spin-echo images that simulate areas of marrow infiltration. Field inhomogeneity can lead not only to failure of fat suppression but to inadvertent saturation of the water peak, which can paradoxically obscure areas of true edema. Short tau inversion recovery is a widely used technique for fat suppression and marrow imaging. With this technique fat is suppressed on the basis of its rapid T1 recovery [6]. The STIR sequences are less affected by field inhomogeneities. The STIR sequences have been shown to have a high sensitivity for the depiction of marrow infiltration [6]. More recently, attempts have been made to characterize bone marrow lesions with MR imaging on the basis of differences in the intracellular fat content of bone marrow [7]. The use of chemical-shift imaging relies on obtaining an image in which fat- and water-resonant peaks are obtained first "in phase" and subsequently "out of phase." This is achieved by altering the echo time (TE). A reduction in signal in the out-ofphase image implies the presence of lipid, whereas an increased or unchanged signal implies its absence. The technique can be useful in identifying the presence of fat coexistent with water in bone marrow lesions. The rationale of this concept is the fact that most neoplastic bone marrow lesions exist as solid masses with progressive destruction and replacement of all lipid and hematopoietic elements.

The use of diffusion imaging for assessing the bone marrow in the foot and ankle, as well as in other body parts, is not yet defined [8]. Although most authorities prefer high-field MR for assessing bone marrow abnormalities of the foot and ankle, the usefulness of low-field MR imaging for this purpose has also been shown [9].

Intravenous administration of gadolinium-based contrast agents is helpful in evaluation for osteomyelitis or malignant marrow infiltration, and in characterization of a cystic or necrotic area. Uptake of gadolinium chelate by the marrow is usually evaluated on T1weighted spin-echo sequences or gradient-echo sequences. A pre-injection series is mandatory since the enhanced signal of the lesion may make it equal to the signal of fatty marrow and thus may render it unapparent. T1-weighted sequences with fat presaturation can be used to render the enhancement more obvious.

\section{Normal bone marrow pattern and variants}

The foot and ankle are targets of progressive conversion of hematopoetic (red) marrow to fatty (yellow) marrow beginning in early childhood. In a newborn, almost all marrow is red marrow with the exception of epiphysis and apophysis [10]. In the foot conversion of red marrow to yellow marrow begins with the phalanges and progresses rapidly to the axial skeleton $[4,11,12,13]$.
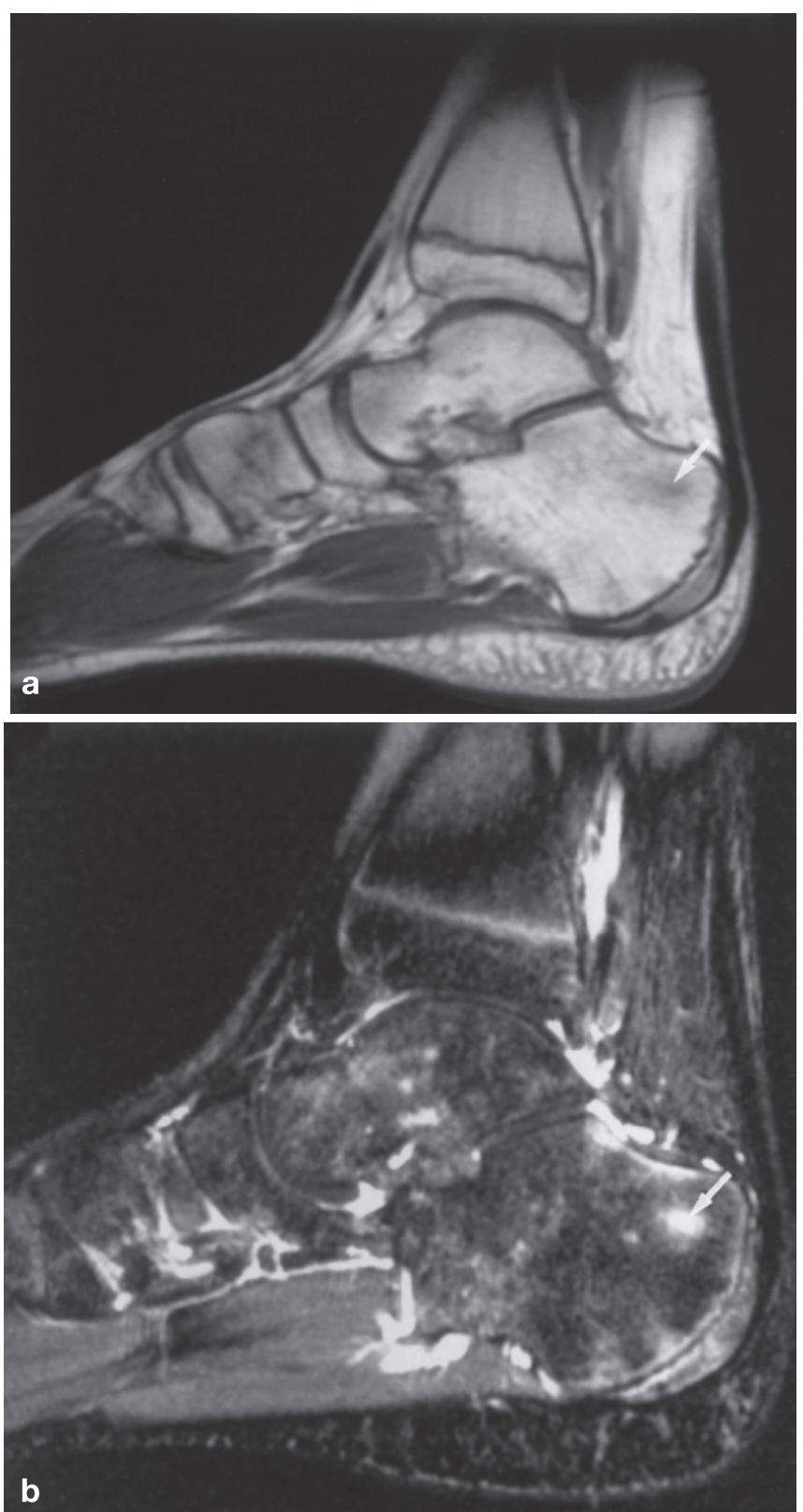

Fig. 1a, b A 13-year-old boy with incomplete marrow conversion. a Sagittal T1-weighted spin-echo image (TR/TE: 450/14 ms) demonstrates multiple small foci and one more larger focus (arrow) of low signal intensity throughout the tarsal bones and calcaneus. b The small foci as well as the larger focus (arrow) are hyperintense (higher signal intensity than muscle) on a sagittal fast short tau inversion recovery (STIR) image (TR/TE: 3880/38 ms; TI: $150 \mathrm{~ms}$ ) consistent with residual islands of red bone marrow

Marrow conversion occurs first in the distal phalanges, epiphysis, and apophysis. By the age of 5 years marrow conversion is nearly complete in the foot and ankle [4]; however, isolated islands of red marrow may persist in the yellow marrow as late as 15 years of age [14]. Re- 

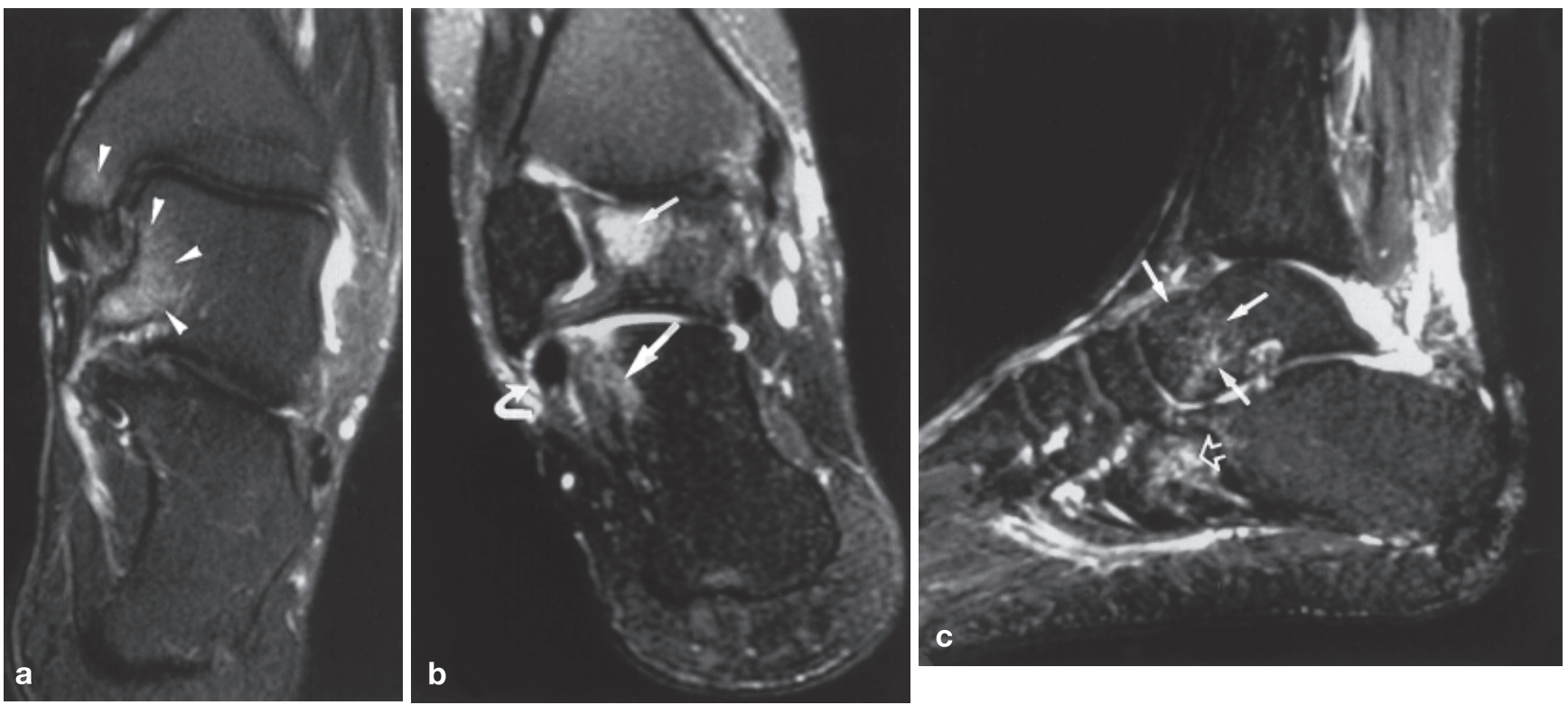

Fig. 2a-c Bone bruises following lateral ankle sprain. a Contralateral bone bruise due to direct impaction injury in a 29-yearold American football player with a lateral ankle sprain and subacute tear of the anterior talofibular ligament (not shown). Axial T2-weighted fat-suppressed fast spin-echo image (TR/TE: 3067/ $89 \mathrm{~ms}$ ) demonstrates marrow edema predominantly in the medial aspect of the talus as well as in the medial malleolus (arrowheads). b Subacute microavulsion injury of the calcaneo-fibular ligament in a 24-year-old woman. Coronal fat-suppressed T2weighted fast spin-echo image (TR/TE: 2967/79 ms) demonstrates absence of the calcaneo-fibular ligament with fluid (curved arrow) surrounding the peroneal tendons. Marrow edema (long arrow) is seen at the insertion of the torn calcaneo-fibular ligament consistent with a microavulsive injury. Marrow edema in the talar neck (short arrow) resulted from an additional occult fracture. c A 31year-old patient with a bone bruise of the talar neck secondary to a rotational injury. Subtle reactive marrow edema (arrows) in the trabecular bone without extension to the cortex is demonstrated as high signal intensity abnormality on sagittal fast STIR image (TR/TE: $4717 / 39 \mathrm{~ms}$; TI: $160 \mathrm{~ms}$ ) consistent with a bone bruise. Posttraumatic marrow edema is seen in the cuboid bone (open arrow)

sidual red bone marrow has a pattern of multiple small foci or confluent areas of high signal intensity particularly conspicuous on STIR sequences; however, STIR sequences may be difficult to interpret in children with cancer since high signal intensity lesions may represent both hematopoietic bone marrow or metastatic lesions. Heterogeneous signal is also seen on T1-weighted images, where the residual red marrow is visible as areas of intermediate signal intensity (higher than muscle but lower than fluid) compared with the high-signal background of yellow marrow. This phenomenon is especially useful in children older than 10 years (Fig. 1). Marrow conversion is often symmetrical, and the tibial epiphysis, talar neck, calcaneus, tarsal joints, and especially the base of the fifth metatarsal bone may contain residual red marrow.

\section{Bone bruise}

Bone bruises represent trabecular disruption of the cancellous bone with hemorrhage and edema extending within the medullary space. Bone bruises resolve without sequelae almost always within 8 weeks and invariably within 12 weeks. Bone bruise of the foot and ankle is uncommon, and is most often a sequelae of ankle sprain [15]. Bone bruises are best demonstrated on T2weighted fat-suppressed or STIR images. Bone bruises may be difficult to detect on non-fat-suppressed MR images. Usually, there is only subtle marrow abnormality on T1-weighted images. Pattern and location of marrow edema in bone bruises may serve as an indicator of the underlying injury vector [15]. If marrow edema is located at the contralateral aspect of the ligamentous tear, a direct impaction should be considered (Fig. 2). Marrow changes at the site of the ligament attachment are caused by a microavulsion injury (Fig. 2). Following a rotational injury, ankle bone marrow edema may be seen in the talar neck (Fig. 2). Direct impact bone bruises are most frequent in the navicular.

\section{Occult fractures}

The term "occult fracture" is used for a fracture initially not seen by radiography. These fractures are still termed "occult" even when confirmed by other imaging tests, or if the fracture is seen in retrospect. Magnetic resonance 

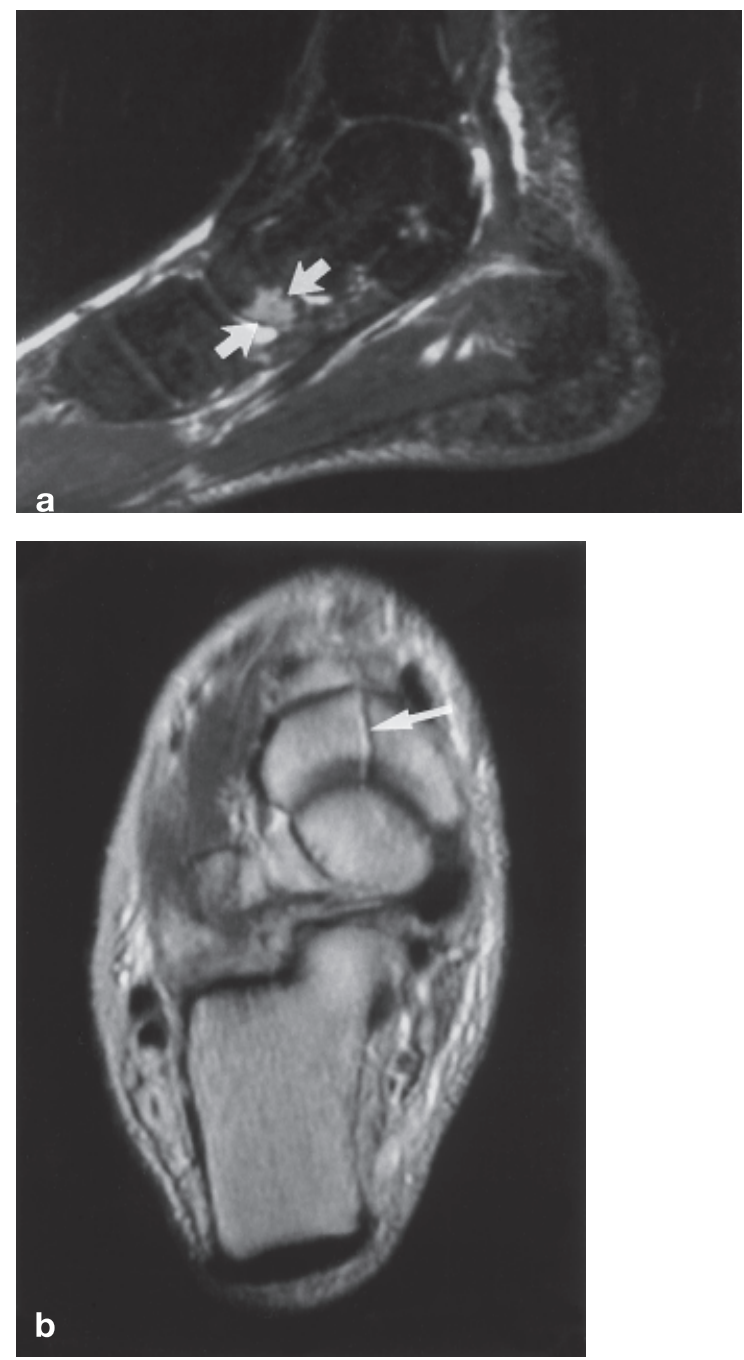

Fig.3a, b Occult fracture of the navicular bone in a 44-year-old male. a Sagittal fast STIR image (TR/TE: 4700/33 ms; TI: $160 \mathrm{ms)}$ shows patchy high signal within the marrow of the navicular bone (arrows). b On intermediate-weighted axial fast spin-echo image (TR/TE: $3800 / 28 \mathrm{~ms}$ ) a fracture line (arrow) is visible

imaging is an excellent test for the presence of an occult fracture since it is extremely sensitive and more specific than scintigraphy $[16,17]$. The MR characteristics of occult fracture are those of poorly defined or speckled areas of bone marrow edema with high signal intensity on fat-suppressed T2-weighted or STIR images (Fig. 3). In contrast to bone bruises, marrow changes of occult fractures are also present on T1-weighted MR sequences [16]. A fracture line is often, but not invariably, seen [16]. The most common locations for occult fractures are navicular, talus, and calcaneus. Although occult fractures in the talus may have variable appearances, they usually extend through the subtalar joint posteriorly to some degree $[18,19]$.

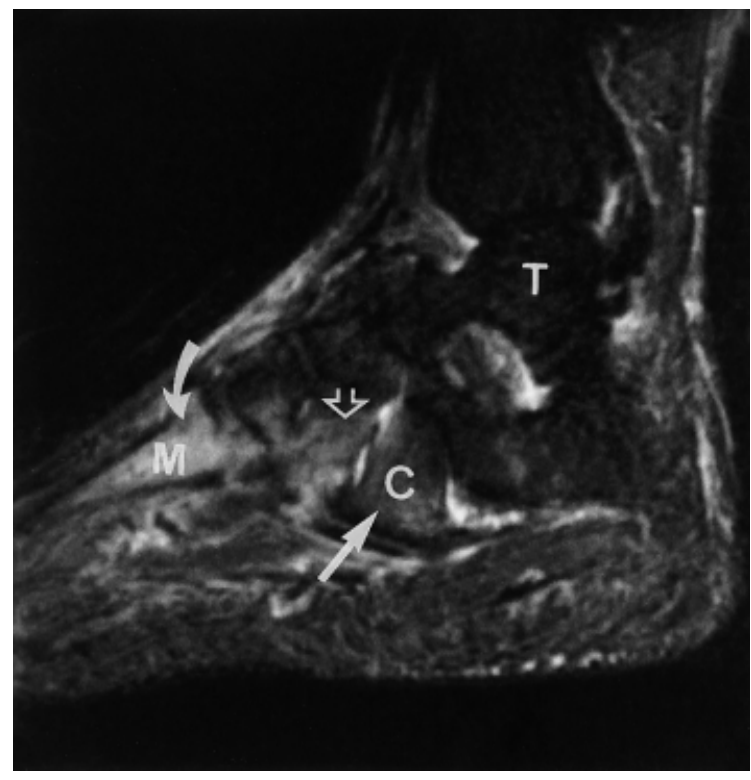

Fig. 4 A 37-year-old female patient with a metatarsal stress fracture and marrow edema due to altered mechanics. Sagittal fast STIR image (TR/TE: 2500/27 ms; TI: $150 \mathrm{~ms}$ ) shows extensive marrow edema in the first metatarsal bone (curved arrow). Subtle marrow edema is also seen in the cuneiforms (open arrows) as well as in the cuboid (arrow). Clinical findings of absent pain at the cuboid and cuneiform are consistent with bone marrow alterations due to altered biomechanics. $C$ cuboid; $T$ talus

\section{Stress injury}

Stress injury is a continuum from the normal response to altered mechanics, to the symptomatic stress response, and eventually to a stress fracture.

\section{Altered mechanics}

Altered weight-bearing due to ambulation or internal derangements in the setting of painful conditions of a painful ankle may result in patchy, ill-defined marrow edema best seen on STIR images [20]. The MR images can show the source of altered mechanics which, for example, may represent an acute tendon tear elsewhere in the foot which is causing the patient to their weightbearing. Edema due to altered mechanics is generally without pain at the site of the abnormal signal and resolves spontaneously (Fig. 4) [20].

\section{Stress response and stress fractures}

Stress response may be considered as a subclinical stress fracture caused by an overuse syndrome [16]. Intramedullary, MR changes consist of patchy loss of marrow fat on T1-weighted sequences and ill-defined hyperin- 


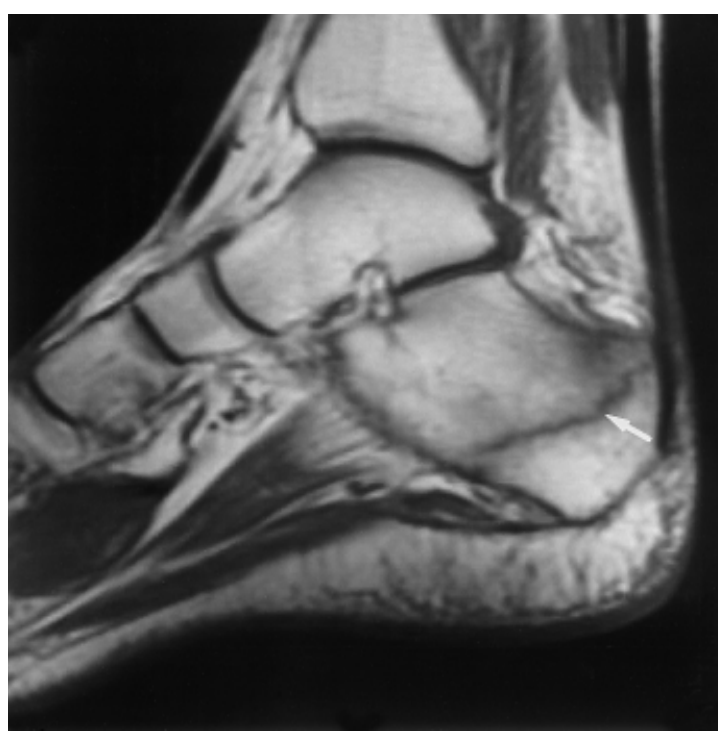

Fig.5 A 21-year-old runner with a stress fracture of the calcaneus. Sagittal T1-weighted spin-echo image (TR/TE: 550/14 ms) demonstrates a fracture line (arrow) with adjacent marrow edema

tensity on STIR sequences [16]. A fracture line is not seen.

Stress fractures may occur in bone that is subjected to multiple episodes of stress, none of which is intense enough to cause an acute fracture. They result from rapid adaptation of muscles to stress and relatively slower adaptation of bones to stress. Stress fractures in the foot and ankle may or may not show a visible fracture line. Often a wide area of marrow edema is seen with adjacent periosteal reaction.

Stress fractures are much more common in the lower extremity than in the upper extremity (Fig. 5). The most common location for a stress fracture is the metatarsals, particularly the second and the third. Other typical locations for stress fractures include the calcaneus and navicular. Occasionally, stress fractures can be occult radiographically, but more often they are suspected and subsequently confirmed by MR imaging. Stress fractures diagnosed initially on MR imaging are mainly those in the intrinsic bones of the tarsus itself, most commonly in the calcaneus followed by fractures of the talus and the navicular $[21,22,23]$. Stress fractures have MR imaging appearance identical to occult fractures.

\section{Subtendinous bone marrow edema}

Occasionally, a variable-sized focus of marrow edema may be seen adjacent to a disordered tendon on T2weighted or STIR images. Subtendinous marrow edema may occur anywhere along the course of the disordered tendon and is most frequently seen with traumatic or

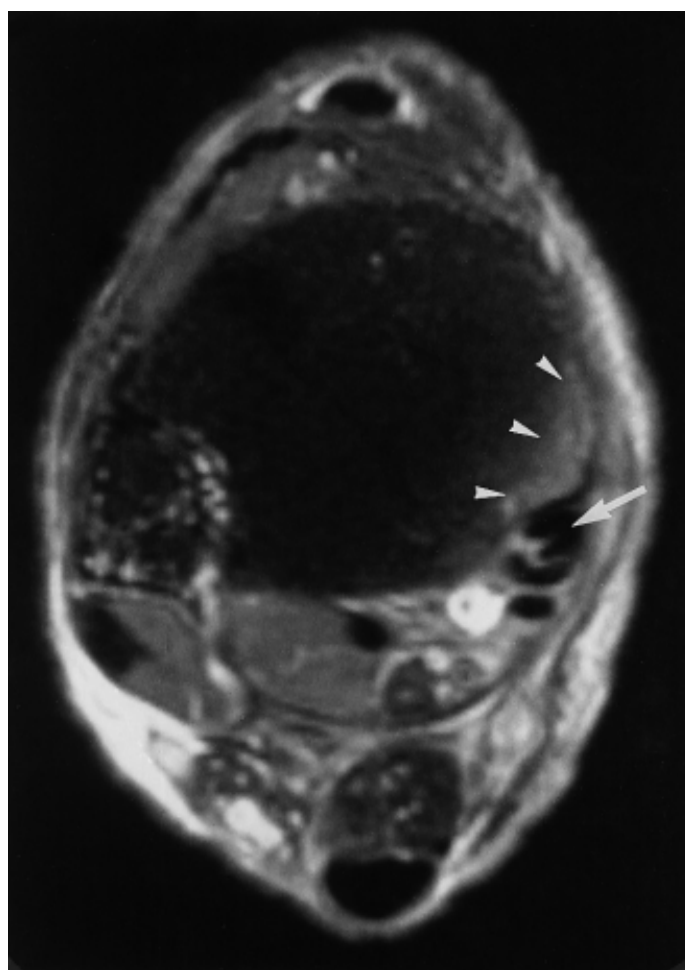

Fig. 6 A 50-year-old woman with chronic tendinosis and an interstitial tear of the posterior tibial tendon (arrow) as well as subtendinous bone marrow edema caused by the tendon disorder. Marrow edema (arrowheads) adjacent to the tendon is seen on axial T2-weighted fat-suppressed fast spin-echo MR image (TR/TE: $5000 / 40 \mathrm{~ms}$ )

degenerative disorders of the posterior tibialis or peroneal tendons or any tendon or bursa involved in an inflammatory arthropathy (Figs. 6, 7) [24, 25]. The cause of this marrow edema remains unclear. It may be related to marrow hyperemia and related to tendon friction or it may be a result of hyperemia of the overlaying tendon sheath. Subtendinous marrow edema may be present at the medial malleolus associated with posterior tibialis tendon abnormalities, and at the calcaneus associated with the peroneus longus and brevis tendons, and at the cuboid associated with the peroneus longus tendon [24]. Subtendinous marrow edema has been shown to be associated with ankle pain medially [24]. When subtendinous marrow edema is present at the Achilles tendon insertion, it is usually caused by an inflammatory arthropathy [25].

\section{Avascular necrosis}

In the foot and ankle avascular necrosis (AVN) is most commonly seen following a fracture of the talus, navicular bone, or base of the fifth metatarsal bone [26]. 


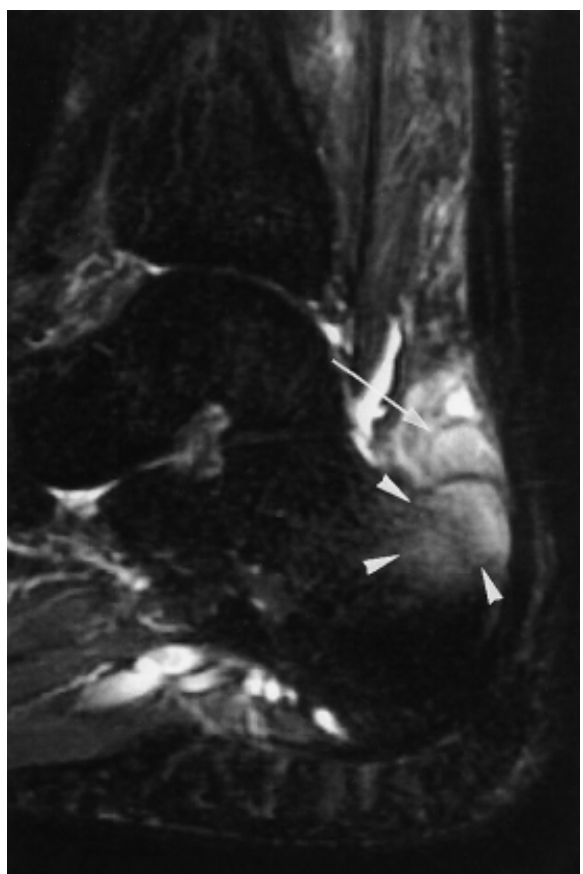

Fig. 7 A 28-year-old male patient with rheumatoid arthritis. Sagittal fast STIR image (TR/TE: 3933/40 ms; TI: $150 \mathrm{~ms}$ ) demonstrates marrow edema at the insertion of the Achilles tendon on the calcaneal tuberosity (arrowheads). In addition, bursitis of the retrocalcaneal Achilles bursa (arrow) is noted

Avascular necrosis is rare following calcaneal fractures and is rare following other metatarsal fractures. The most common systemic disease that causes AVN in the foot and ankle is systemic lupus erythematosis [16]. Other causes of systemic diseases causes AVN in the foot and ankle include alcoholism, Cushing's syndrome and exogenous administration, pregnancy, Caisson disease, alcoholism, and pancreatitis [16].

The bone marrow signal in AVN is characteristically of low signal intensity on T1-weighted images, which may or may not increase on T2-weighted MR images. Avascular necrosis displays a low signal intensity on T2weighted images once the lesion shows sclerosis on plain radiographs. The characteristic "double-line sign" of AVN is less frequent in the foot and ankle [16]; however, if the double-line sign is present, systemic disorders should be considered because most post-traumatic AVN lack the classic double-line sign (Fig. 8). Systemic causes of AVN have a predilection for the talus and the calcaneus.

\section{Osteochondral defects}

Osteochondral defects (OCD) of the foot and ankle are most commonly seen in the talar dome and may involve

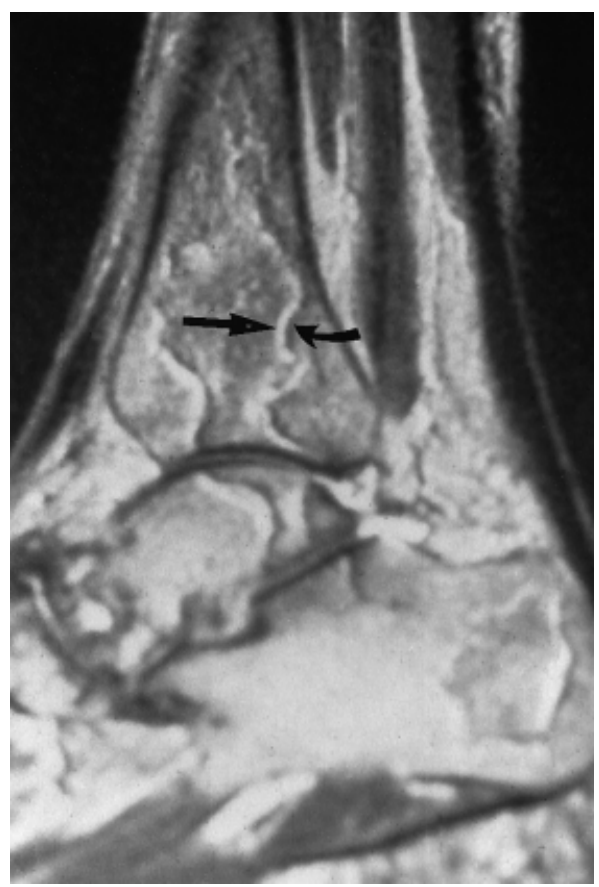

Fig. 8 A 39-year-old patient with systemic lupus erythematosis and avascular necrosis. Characteristic "double-line sign" on sagittal fat-suppressed T2-weighted fast spin-echo image (TR/TE: 7800/ $90 \mathrm{~ms}$ ) of the ankle with a high-signal-intensity band in the inner zone (arrow) and a low-signal-intensity band in the parallel outer zone (curved arrow)

the medial or lateral aspect of the talus [16]. Osteochondral defects laterally are typically seen at or slightly anterior to the equator of the talus; medially, they are seen posterior to the equator of the talus. If an OCDtype lesion is seen in another location, subchondral cysts or geodes should be suspected. The lesions are typically the result of either a single episode of trauma or repetitive minor trauma.

The staging of OCD is determined by whether the overlying cartilage layer is intact $[27,28,29]$. This is difficult to determine and requires high-resolution MR imaging. Sometimes direct or indirect MR arthrography is necessary. There is limited published experience with intravenous gadolinium-enhanced scans to evaluate OCD in the ankle. The classification system is also based whether there is a rim of high signal on T2weighted images around the lesion [27, 28, 29]. If the high-signal rim is partial, it is a partially loose OCD; if the rim of high signal intensity is complete, it is a loose OCD. This rim, although bright on T2-weighted images, may represent granulation tissue and not fluid. If a cyst is seen underneath the OCD, the defect is invariably loose regardless of what the rim looks like (Fig. 9). Lastly, if marrow edema is seen about an OCD, it is at least loose in situ. 


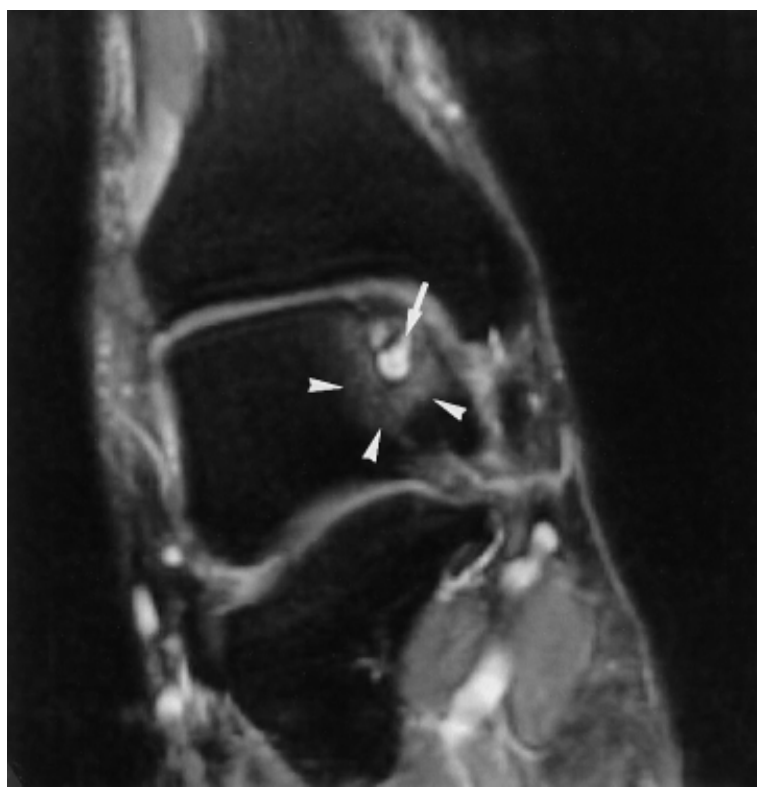

Fig.9 A 45-year-old patient with a loose osteochondral defect at the talar dome. Coronal fat-suppressed T2-weighted image (TR/ TE: 7800/90 ms) demonstrates subchondral cystic changes (arrow) at the medial aspect of the talar dome with surrounding marrow edema (arrowheads)

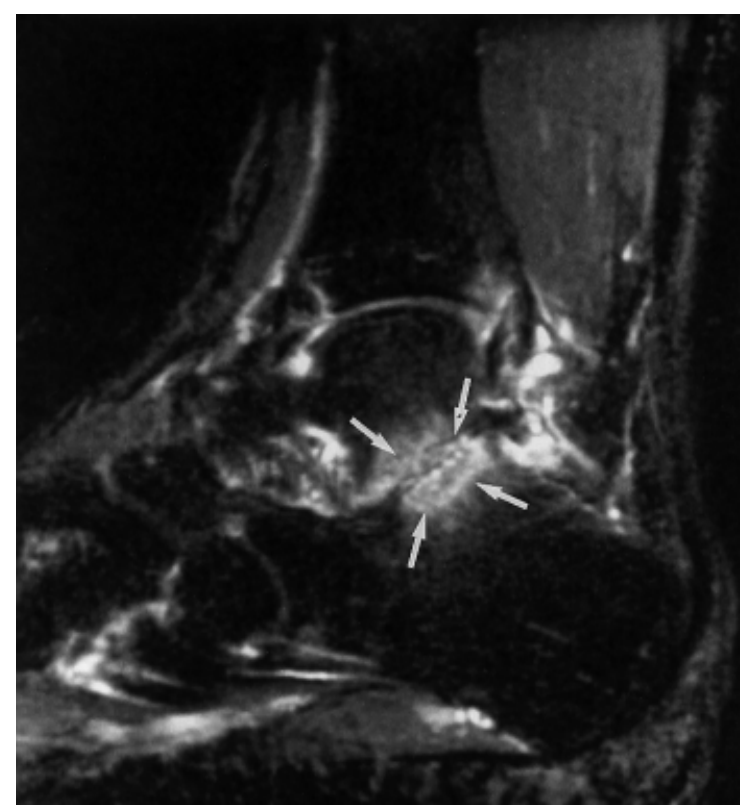

Fig.10 Posttraumatic subtalar osteoarthritis with reactive marrow edema in a 48-year-old male patient. Sagittal fast STIR image (TR/ TE: 4715/39 ms; TI: $160 \mathrm{~ms}$ ) shows "kissing" bone marrow edema on both sides of the subtalar joint space (arrows) associated with joint space narrowing. Slight osteoarthritis is also present in the tibio-talar joint
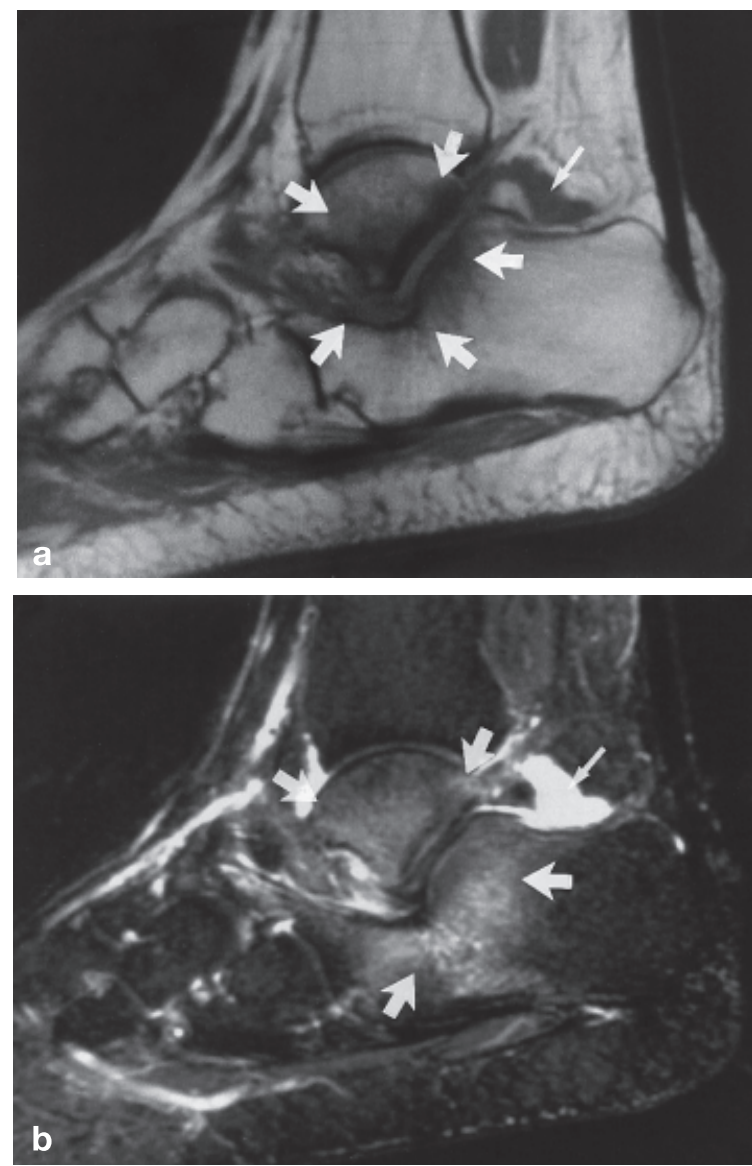

Fig. 11 a, b A 39-year-old patient with early rheumatoid arthritis. a Sagittal T1-weighted spin-echo image (TR/TE: 600/16 ms) and b sagittal fast STIR image (TR/TE: $3933 / 40 \mathrm{~ms}$; TI: $150 \mathrm{~ms}$ ) show extensive bone marrow edema on both sides of the subtalar joint (thick arrows). Marrow edema without the presence of osteophytes and joint space narrowing suggests inflammatory arthropathy. Increased amount of ankle joint fluid in the posterior ankle joint recess is noted (thin arrows)

\section{Osteoarthritis}

Osteoarthritis involves articular cartilage and subsequently subchondral bone. Metatarsophalangeal joints are commonly affected. Less frequently, the subtalar and ankle joints are affected. Due to cartilage loss and joint-space narrowing, reactive marrow edema with subchondral cyst formation is typically present on both sides of the joint giving the appearance of "kissing" lesions (Fig. 10). This is a useful sign since direct visualization of cartilage defects is quite difficult. 

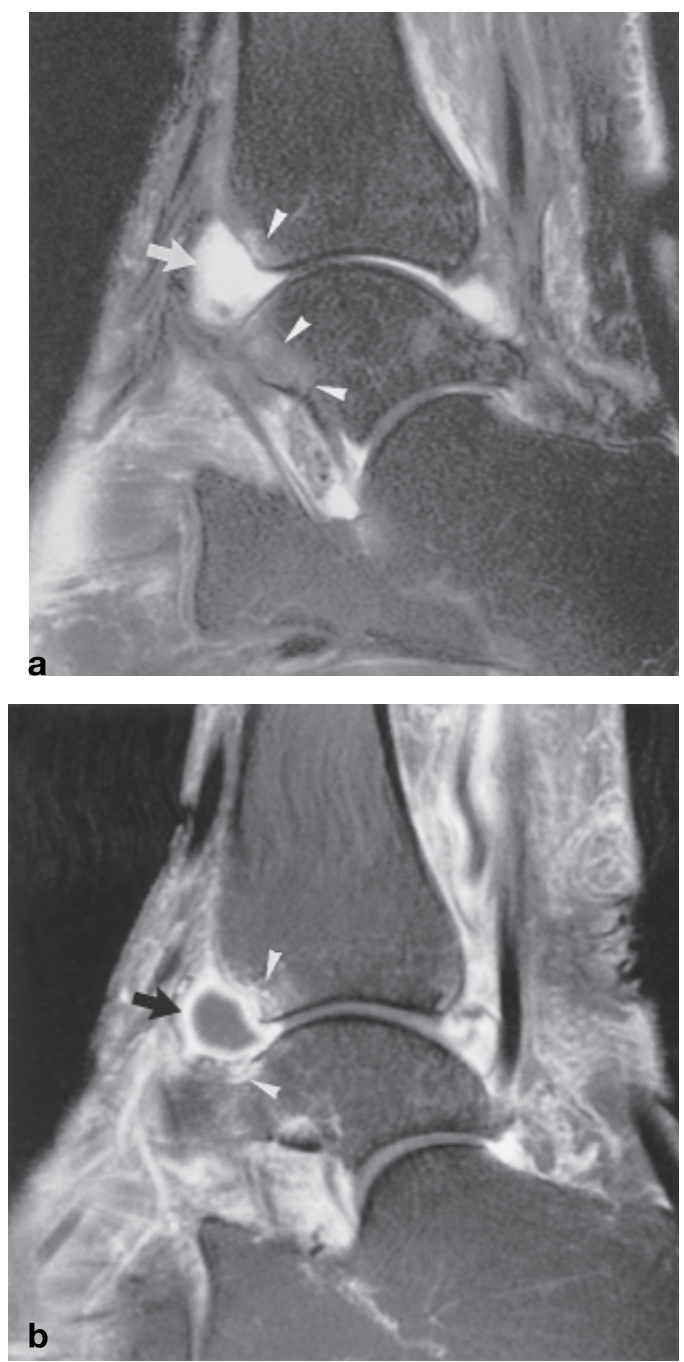

Fig. 12a, b A 45-year-old patient with septic arthritis of the ankle joint. a Sagittal T2-weighted fast spin-echo image (TR/TE: 3766/ $80 \mathrm{~ms}$ ) demonstrates effusion in the ankle joint (arrow), a small focus of juxta-articular marrow edema (arrowheads), and soft tissue swelling. b After intravenous contrast administration, synovial enhancement (arrow) and slight enhancement of the adjacent bone marrow (arrowheads) is seen on a sagittal fat-suppressed T1weighted MR image (TR/TE: 767/17 ms)

\section{Inflammatory arthropathies}

The foot and ankle are frequently involved by inflammatory arthropathies such as rheumatoid arthritis, Reiter's disease, and psoriasis [30]. Presence of marrow edema is typical for inflammatory arthropathies such as rheumatoid arthritis; therefore, it is helpful to know the target areas for various inflammatory arthropathies. For example, rheumatoid arthritis typically involves the metatarsophalangeal joints, the subtalar joint, the talonavicular, and somewhat less frequently involves the
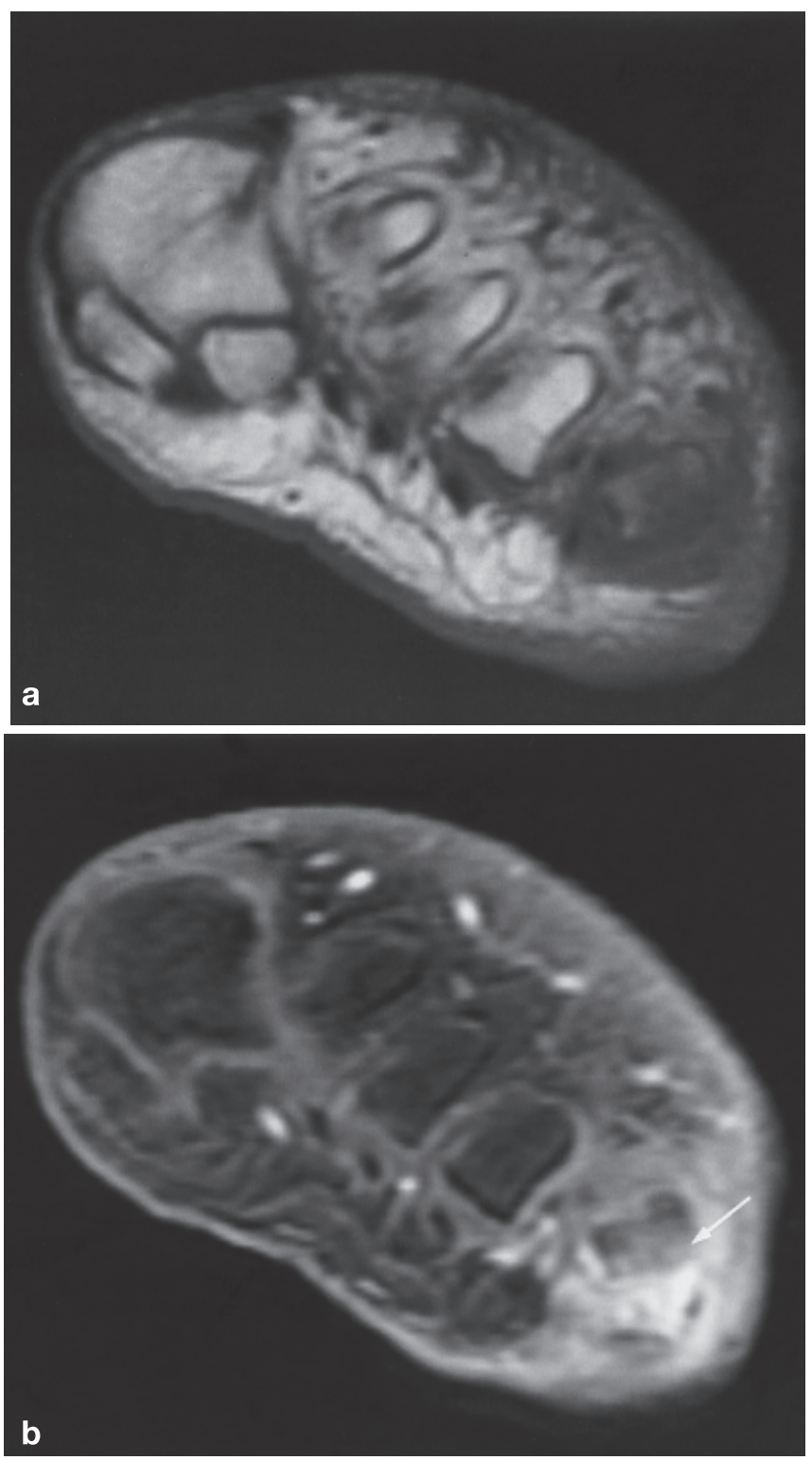

Fig.13 a, b A 65-year-old patient with osteomyelitis of the fifth metatarsal head. a Axial T1-weighted spin-echo image (TR/TE: $600 / 16 \mathrm{~ms}$ ) shows marrow edema and soft tissue swelling of the fifth metatarsal. b T1-weighted spin-echo image after administration of gadolinium-based contrast agent demonstrates enhancement of both bone marrow and cortical bone (arrow) as well as enhancement of surrounding tissue

ankle joint and the intertarsal joint [31]. Psoriasis tends to have a ray-like distribution with extensive soft tissue reactive changes all the course of a ray or a digit, and less prominent articular disease [32]. Reiter's disease often causes a periosteal reaction at the malleoli, particularly medially, and about the metatarsals, particularly the fifth metatarsal bone [33]. 

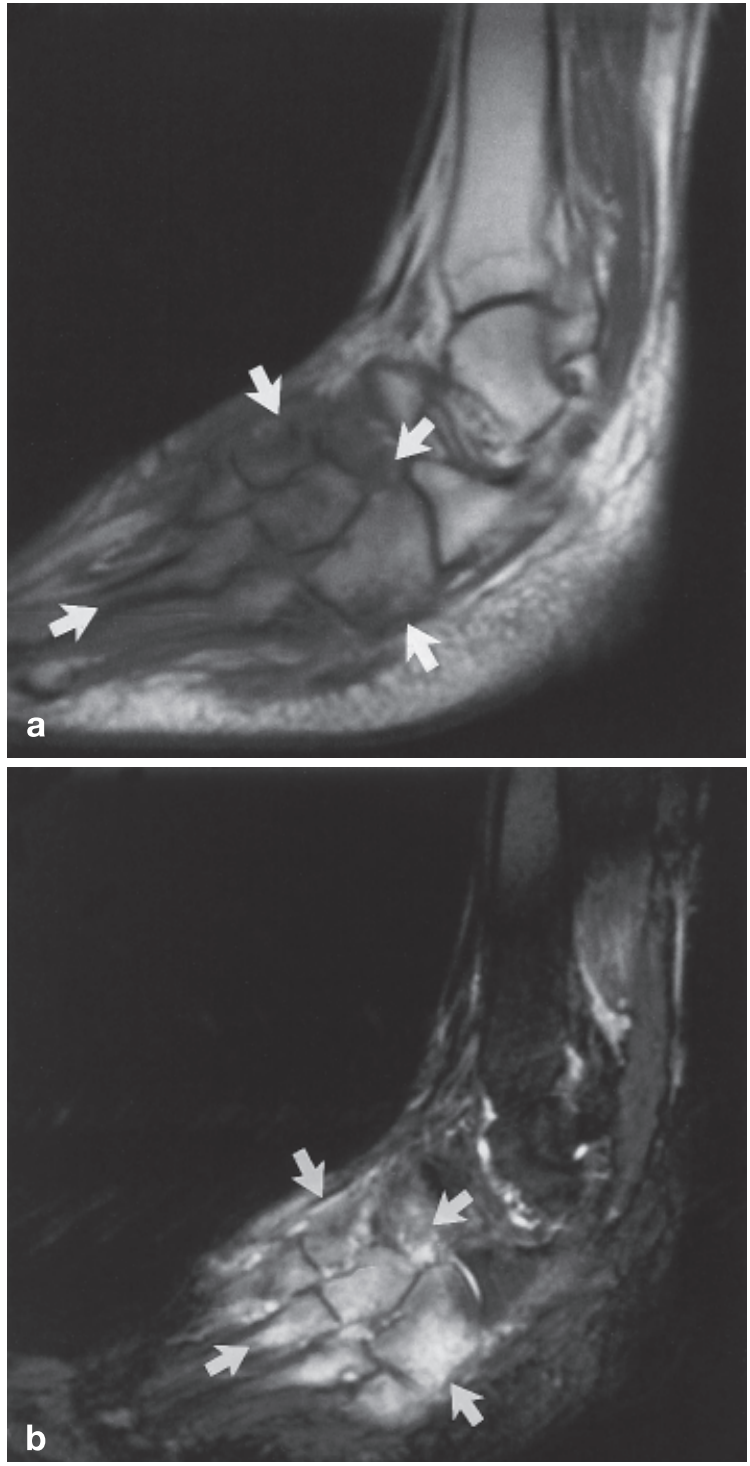

Fig. 14a, b A 35-year-old female patient with diabetes mellitus in an early stage of neuropathic osteoarthropathy. a Sagittal T1weighted spin-echo (TR/TE: 600/14 ms) image demonstrates extensive replacement of normal fatty marrow (arrows) by low signal intensity at the level of Lisfranc joints and midfoot. b On a corresponding sagittal fast STIR image (TR/TE: 4700/39 ms; TI $160 \mathrm{~ms}$ ) abnormal marrow is bright (arrows). The bones are preserved in shape and alignment. Location and pattern of the marrow edema and lack of destructive changes suggest an early stage of neuropathic osteoarthropathy

Extensive subchondral marrow edema without osteophytes is mostly located in the talonavicular or in the subtalar joint and is a frequent finding in early stage of rheumatoid with involvement of the foot. Joint effusion is often seen in patients with inflammatory arthropathies involving the foot and ankle (Fig. 11).
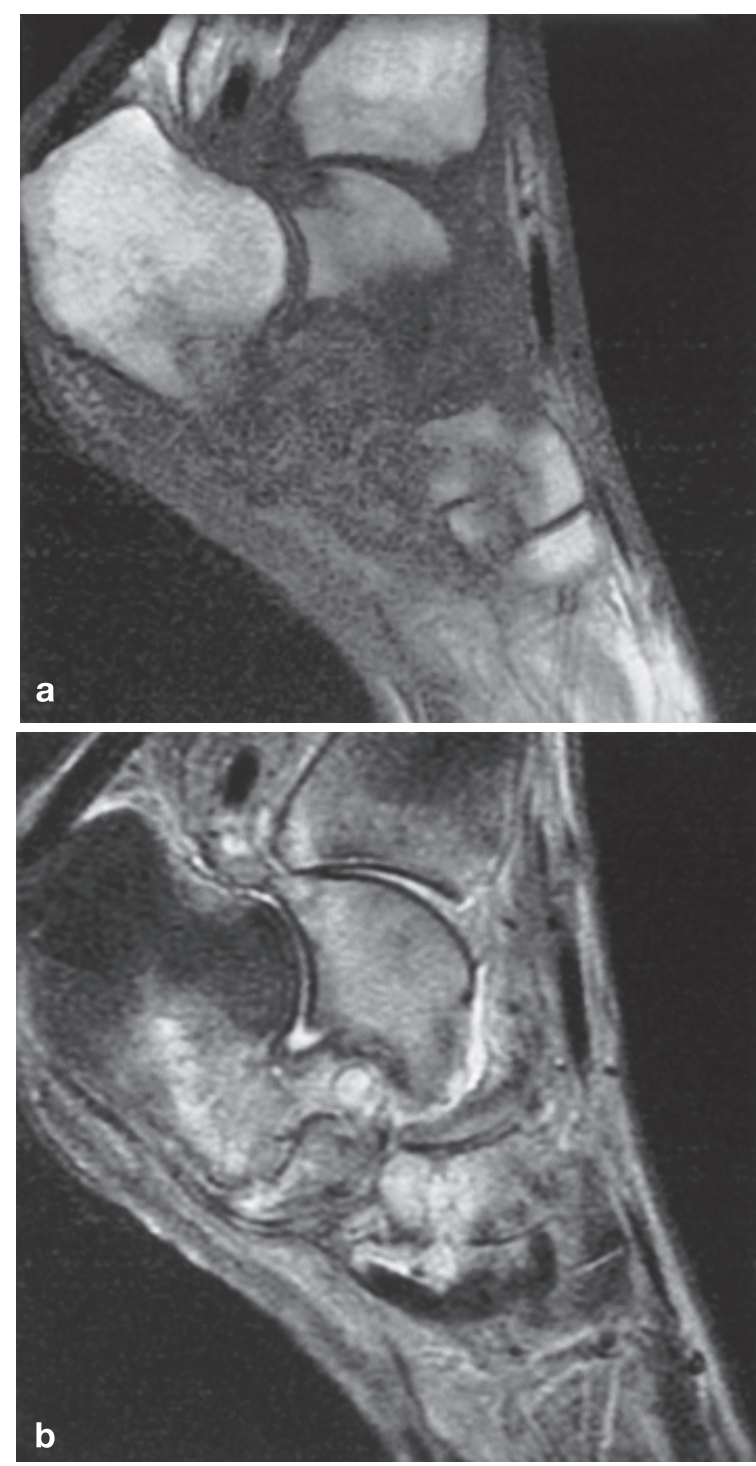

Fig. 15a, b A 67-year-old patient with chronic neuropathic osteoarthropathy and no history of superimposed infection. a Sagittal T1-weighted spin-echo image (TR/TE: 500/20 ms) demonstrates decreased signal intensity within the marrow of the amorphic talar neck, anterior calcaneal process as well navicular and cuboid bones. b Disorganization, fragmentation, and marrow edema are visible on corresponding sagittal fast STIR image (TR/TE: 4700/39 ms; TI: $160 \mathrm{~ms}$ ). The bones of the midfoot are not delineated in their full extent, representing a negative "ghost sign." This is a characteristic finding in chronic neuropathic osteoarthropathy. In addition, soft tissue swelling is noted

\section{Septic arthritis}

Septic joints show an increased amount of joint fluid and edema in the adjacent soft tissues. The effusions in septic joints are often of higher signal intensity than that of simple fluid on T1-weighted sequences. This occurs 
because of the proteinaceous content of infectious fluid, causing higher signal on T1-weighted sequences. The synovium in septic arthritis is thickened and enhances intensely following intravenous administration of gadolinium-based contrast agents. In patients with septic arthritis small juxta-articular focal reactive bone marrow edema is present which may be confused with osteomyelitis (Fig. 12). In true bone infection the bone marrow edema is more extensive than this reactive edema, involving not only the subchondral bone but also central medullary bone.

\section{Osteomyelitis}

Osteomyelitis of the foot and ankle is usually a result of contiguous spread. The MR diagnosis of osteomyelitis is based mainly on primary signs which represent replacement of fatty marrow by edema, infiltration by inflammatory cells, hemorrhage, fibrin, and debris. Areas of replaced marrow are seen as loss of fatty marrow signal on T1-weighted images with enhancement following intravenous administration of gadolinium-based contrast agent (Fig. 13) [34]. If there is no enhancement, either a sequestrum is present or the bone is devitalized secondary to poor vascularity [35]. On fat-suppressed T2weighted or STIR images increased marrow signal representing marrow edema is usually present in osteomyelitis. Since most cases of osteomyelitis in the foot and ankle are secondary to adjacent soft tissue infection, almost always ulcerations or sinus tracts are demonstrated. If sinus tract and ulceration is adjacent to the marrow abnormality, osteomyelitis can be confidently diagnosed.

In certain pathological conditions, such as neuropathic osteoarthropathy and biomechanical stress changes, these primary signs may be equivocal $[36,37]$. In these cases secondary signs adjacent to a marrow abnormality, including cutaneous ulcer, cellulitis, soft tissue mass, abscess, sinus tract, and cortical interruption, are extremely helpful for diagnosis of complicating osteomyelitis $[36,37]$.

\section{Neuropathic arthropathy}

Neuropathic osteoarthropathy can affect various joints in the foot, particularly the Lisfranc and Chopart joints. Radiographically neuropathic arthropathy is characterized by joint debris, joint dislocation, joint disorganization, and preserved bone density [37]. Extensive marrow edema may be seen with neuropathic arthropathy. In an early acute stage (preceding typical destructive chronic changes), diffuse, extensive areas of marrow edema secondary to microtrauma or cartilagenous fibrillation and fragmentation is seen (Fig. 14).

The diagnosis of osteomyelitis in a neuropathic foot is notoriously difficult due to the frequently concomitant and often confounding arthropathy. Three basic signs may be used to differentiate neuropathic arthropathy from infection. Firstly, if the marrow abnormalities are adjacent to a sinus tract or ulceration presence of osteomyelitis is more likely [35]. The second sign is location. Neuropathic osteoarthropathy typically involves the midfoot and Lisfranc joints and infection occurs secondary to adjacent trauma sites or skin abrasion. In addition, soft tissue changes are more extensive in infection than those of neuropathic arthropathy. Perhaps the most specific sign in this setting is the "ghost" sign [38]. The ghost sign is characterized by a loss of definition of the individual bones (usually tarsal) on T1weighted images. On STIR or following gadolinium injection, the normal individual outline becomes evident. The ghost sign is absent in neuropathic arthropathy, because the loss of definition on T1-weighted images is due to true fragmentation and disorganization (Fig. 15). Conversely, the "ghost sign" is positive in osteomyelitis.

\section{Conclusion}

In this review, various aspects of MR imaging of the foot and ankle including normal variants of the bone marrow of the foot and ankle, as well as the varied responses of bone marrow to trauma, stress, and disease have been discussed. Knowledge about the different patterns of bone marrow signal abnormalties may help distinguish the various types of marrow disorders of the foot and ankle.

\section{References}

1. Jones KM, Unger EC, Granstrom P, Granstrom P, Seeger JF, Carmody RF, Yoshino M (1992) Bone marrow imaging using STIR at 0.5T and 1.5T. Magn Reson Imaging 10: 169-176
2. Vande Berg BC, Malghem J, Lecouvet FE, Maldague B (1998) Magnetic resonance imaging of the normal bone marrow. Skeletal Radiol 27: 471-483

3. Vande Berg BC, Malghem J, Lecouvet FE, Maldague B (1998) Eur Radiol 8: 1327-1334
4. Steiner RM, Mitchell DG, Rao VM, Schweitzer ME (1993) Magnetic resonance imaging of diffuse marrow disorders. Radiol Clin North Am 31: 383-409 
5. Peterfy CG, Linares R, Steinbach LS (1994) Recent advances in magnetic resonance imaging of the musculoskeletal system. Radiol Clin North Am 32: 291-311

6. Fleckenstein JL, Archer BT, Barker BA et al. (1991) Fast-short tau inversionrecovery MR imaging Radiology 197: 499-504

7. Disler DG, McCauley TR, Ratner LM, Kesack CD, Cooper JA (1997) In-phase and out-of-phase MR imaging of bone marrow: prediction of neoplasia based on the detection of coexistent fat and water. AJR 169: 1439-1447

8. Vanel D, Dromain C, Tardivon A (2000) MRI of bone marrow disorders. Eur Radiol 10: 224-229

9. Hottya GA, Peterfy CG, Uffmann M, Häckl FO, Le Hir P, Redei J, Gindele AU, Dion E, Genant HK (2000) Dedicated extremity MR imaging of the foot and ankle. Eur Radiol 10: 467-475

10. Vogler JB, Murphy WA (1988) Bone marrow imaging. Radiology 168 : 679-693

11. Dooms GC, Fisher MR, Hricak H, Richardson M, Crooks LE, Genant $\mathrm{H}$ (1985) Bone marrow imaging: magnetic resonance studies related to age and sex. Radiology 155: 429-443

12. Weinreb J (1990) MR imaging of bone marrow. A map would help. Radiology 177: $23-24$

13. Taccone A, Oddone M, Dell'Acqua A, Occhi M, Ciccone MA (1995) MRI "road map" of normal age-related bone marrow. II. Thorax, pelvis and extremities. Pediatr Radiol 25: 596-606

14. Pal CR, Tasker AD, Ostlere SJ, Watson MS (1999) Heterogenous signal in bone marrow on MRI of children's feet: a normal finding? Skeletal Radiol 28: 274-278

15. Labovitz JM, Schweitzer ME (1998) Occult osseous injuries after ankle sprains. Foot Ankle Int 19: 661-667

16. Schweitzer ME, Karasick D (1994) MR of the ankle and hindfoot. Semin Ultrasound CT MRI 15: 410-422
17. Yao L, Lee JK (1988) Occult intraosseous fracture: detecting with MR imaging. Radiology 167: 749-754

18. Robbins MI, Wilson MG, Sella EJ (1998) MR imaging of anterosuperior calcaneal process fractures. AJR 172: 475-479

19. Sanders TG, Ptaszek AJ, Morrison WB (1999) Fracture of the lateral process of the talus: appearance at MR imaging and clinical significance. Skeletal Radiol 28: 236-239

20. Schweitzer MR, White LM (1996) Does altered biomechanics cause marrow edema? Radiology 198: 851-853

21. Martin SD, Healy JH, Horowitz S (1993) Stress fractures. MRI Orthop 16: 75-78

22. Yao L, Johnson C, Gentili A, Lee JK, Seeger LL (1998) Stress injuries of bone: analysis of MR imaging staging criteria. Acad Radiol 5: 34-40

23. Umans H, Pavlov H (1995) Insufficiency fractures of the talus: diagnosis with MR imaging. Radiology 197: 439-442

24. Morrison WB, Carrino JA, Schweitzer ME, Sanders TG, Raiken DP, Johnson CE (2001) Subtendinous bone marrow edema on MR images of the ankle: association with symptoms and tendinopathy. AJR 176: 1149-1154

25. Golstein-Schainberg C, Homsi C, Pereira RMR, Cossermelli W (1992) Retrocalcaneal bursitis in juvenile arthritis. Ann Rheum Dis 51: 1162-1163

26. Thordarson DB, Triffon MJ, Terk MR (1996) Magnetic resonance imaging to detect avascular necrosis after open reduction and internal fixation of talar neck fractures. Foot Ankle Int 17: 439-442

27. De Smet AA, Fisher DR, Graf BK, Lange RH (1990) Osteochondritis dissecans of the knee: value of MR imaging in determining lesion stability and the presence of articular cartilage defects. AJR 155: 549-553

28. Nelson DW, DiPaola JD, Colville MR, Schmidgall J (1990) Osteochondritis dissecans of the talus and knee: prospective comparison of MR and arthroscopic classification. J Comput Assist Tomogr 14: 804-808
29. DiPaola JD, Nelson DW, Colville MR (1991) Characterizing osteochondral lesions by magnetic resonance imaging. Arthroscopy 7: 101-106

30. Weishaupt D, Schweitzer ME, Alam F, Karasick D, Wapner K (1991) MR imaging of inflammatory joint diseases of the foot and ankle. Skeletal Radiol 28: 663-669

31. Resnick D, Niwayama A (1995) Rheumatoid arthritis. In: Resnick D, Niwayama A (eds) Diagnosis of bone and joint disorders, 3rd edn. Saunders, Philadelphia, pp 866-970

32. Rana NA (1991) Rheumatoid arthritis, other collagen diseases, and psoriasis of the foot. In: Jahss MH (ed) The foot and ankle, 2nd edn. Saunders, Philadelphia, pp 1719-1751

33. Martel W, Braunstein EM, Borlaza G, Good AE, Griffin PE (1979) Radiologic features of Reiter's disease. Radiology 132: $1-10$

34. Morrison WB, Schweitzer ME, Bock GW, Mitchell DG, Hume EL, Pathria MN, Resnick D (1993) Diagnosis of osteomyelitis: utility of fat-suppressed contrast-enhanced MR imaging. Radiology 189: 251-257

35. Morrison WB, Schweitzer ME, Wapner KL, Hecht PJ, Gannon FH, Behm WR (1995) Osteomyelitis in feet of diabetics: clinical accuracy. Surgical utility, and cost-effectiveness of MR imaging. Radiology 196: 557-564

36. Morrison WB, Schweitzer ME, Batte WG, Radack DP, Russel KM (1998) Osteomyelitis of the foot: relative importance of primary and secondary MR imaging signs. Radiology 207: 625-632

37. Deely DM, Schweitzer ME (1997) MR imaging of bone marrow disorders. Radiol Clin North Am 35: 193-212

38. Yuh WTC, Corson J, Baraniewski HM et al. (1989) Osteomyelitis of the foot in diabetic patients: evaluation with plain fild, $99 \mathrm{~m}$ TcMDP, bone scintigraphy and MR imaging. Am J Roentgenol 152: 792-800 